Check for updates

Cite this: RSC Adv., 2018, 8, 19409

Received 25th March 2018 Accepted 14th May 2018

DOI: $10.1039 / c 8 r a 02588 f$

rsc.li/rsc-advances

\section{Poly(allylamine)/tripolyphosphate coacervates enable high loading and multiple-month release of weakly amphiphilic anionic drugs: an in vitro study with ibuprofen $\uparrow$}

\begin{abstract}
Udaka K. de Silva, ${ }^{a}$ Jennifer L. Brown $\star^{a}$ and Yakov Lapitsky (ID *ab
When synthetic polyamines, such poly(allylamine hydrochloride) (PAH), are mixed with crosslink-forming multivalent anions, they can undergo complex coacervation. This phenomenon has recently been exploited in various applications, ranging from inorganic material synthesis, to underwater adhesion, to multiple-month release of small, water-soluble molecules. Here, using ibuprofen as a model drug molecule, we show that these coacervates may be especially effective in the long-term release of weakly amphiphilic anionic drugs. Colloidal amphiphile/polyelectrolyte complex dispersions are first prepared by mixing the amphiphilic drug (ibuprofen) with PAH. Pentavalent tripolyphosphate (TPP) ions are then added to these dispersions to form ibuprofen-loaded PAH/TPP coacervates (where the strongly-binding TPP displaces the weaker-bound ibuprofen from the PAH amine groups). The initial ibuprofen/PAH binding leads to extremely high drug loading capacities (LC-values), where the ibuprofen comprises up to roughly $30 \%$ of the coacervate mass. Conversely, the dense ionic crosslinking of PAH by TPP results in very slow release rates, where the release of ibuprofen (a small, water-soluble drug) is extended over timescales that exceed 6 months. When ibuprofen is replaced with strong anionic amphiphiles, however (i.e., sodium dodecyl sulfate and sodium dodecylbenzenesulfonate), the stronger amphiphile/polyelectrolyte binding disrupts PAH/TPP association and sharply increases the coacervate solute permeability. These findings suggest that: (1) as sustained release vehicles, PAH/TPP coacervates might be very attractive for the encapsulation and multiple-month release of weakly amphiphilic anionic payloads; and (2) strong amphiphile incorporation could be useful for tailoring PAH/TPP coacervate properties.
\end{abstract}

\section{Introduction}

When polyelectrolytes are mixed with polymer-binding multivalent counterions, they can undergo associative phase separation and form either gels, coacervates or precipitates that are rich in both species and coexist with dilute supernatant phases. ${ }^{\mathbf{1 - 4}}$ One class of such systems that has attracted considerable interest is mixtures of synthetic, primary amine-bearing polycations, such as poly(allylamine hydrochloride) (PAH) or poly(L-lysine) (PLL), with multivalent anions - e.g., citrate, phosphate, tripolyphosphate (TPP) and ethylenediaminetetraacetic acid (EDTA). ${ }^{4-8}$ These mixtures form coacervates, which have been explored for

${ }^{a}$ Department of Chemical Engineering, University of Toledo, Toledo, Ohio 43606, USA. E-mail: yakov.lapitsky@utoledo.edu; Fax: +1-419-530-8086; Tel: +1-419-530-8254

${ }^{b}$ School of Green Chemistry and Engineering, University of Toledo, Toledo, Ohio 43606, USA

$\dagger$ Electronic supplementary information (ESI) available. See DOI: 10.1039/c8ra02588f

\$ Current address: Department of Chemical Engineering, University of California, Santa Barbara, California 93106. a variety of applications, ranging from serving as templates for inorganic material synthesis, ${ }^{\mathbf{8}, 9}$ to underwater adhesion, ${ }^{6,7}$ to medical imaging, ${ }^{\mathbf{1 0}}$ photothermal therapy ${ }^{\mathbf{1 1}}$ and controlled release. ${ }^{5,12,13}$

When weaker-binding multivalent anions (such as citrate, phosphate and EDTA) are used, the resulting polymer networks contain short-lived ionic crosslinks and have liquid-like properties. ${ }^{4,6}$ Consequently, since these coacervates flow and (when prepared as macroscopic phases) fail to hold their shape, ${ }^{\mathbf{1 2}}$ they are typically used as colloidal coacervate droplets, which are coated with a stabilizing material that prevents their coalescence. ${ }^{4,5,8}$ Conversely, when strongly binding TPP and pyrophosphate (PPi) ions are used, the ionic crosslinks have longer relaxation times, which result in putty- or gel-like behavior, along with an ability to adhere to a variety of surfaces under wet conditions. ${ }^{6,7}$ This improved ability to hold their shape and remain at their application sites enables the PPi- and TPPcrosslinked coacervates to be broadly used in both colloidal dispersion and macroscopic putty/gel forms. ${ }^{12}$ 
(a)

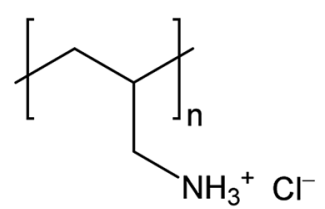

(b)

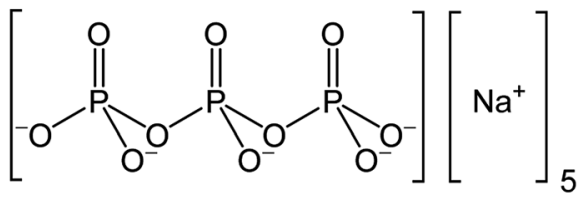

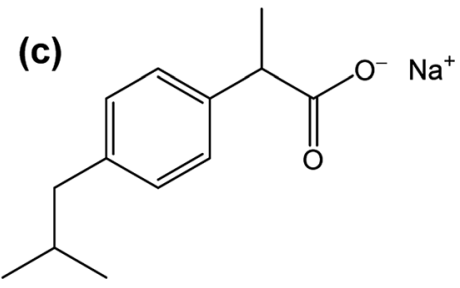

Scheme 1 Chemical structures of (a) PAH and sodium salts of (b) TPP and (c) ibuprofen.

Recently, our group has shown how, by preparing PAH/PPi and PAH/TPP ionic networks, multiple-month release of small, hydrophilic (dye) molecules could be achieved. ${ }^{12}$ These highly extended release times (which evidently reflected the very small hydrodynamic mesh sizes within the densely crosslinked $\mathrm{PAH} / \mathrm{PPi}$ and PAH/TPP networks) were achieved regardless of the payload/polymer network affinity. ${ }^{12}$ Moreover, these release times were much longer than those normally obtained with hydrogels where, without encapsulating the payload inside dispersed/release rate-controlling colloids (e.g., liposomes, microgels or nanoparticles), ${ }^{14-19}$ covalently conjugating the payload to the base polymer molecules, ${ }^{20-22}$ or relying on solutespecific gel/payload binding interactions, ${ }^{23,24}$ release of small water-soluble drug molecules cannot typically be sustained over many days, weeks or months. ${ }^{25}$ Additionally, these PAH-based complexes (especially the PAH/TPP complex) show promising in vitro cytocompatibility. ${ }^{12}$ Given that $\mathrm{PAH}$ is already being used in its covalently crosslinked form as an oral drug, ${ }^{26}$ and both PPi and TPP are on the U.S. Food and Drug Administration's generally regarded as safe (GRAS) list, these findings suggest that PAH/PPi and PAH/TPP coacervates may prove to be attractive vehicles for long-term drug delivery. ${ }^{12}$

Building on this previous work, here we explore the use of gel-like coacervates prepared by crosslinking PAH with TPP for the encapsulation and long-term release of the antiinflammatory drug, ibuprofen (see Scheme 1). Besides the potential utility of this drug/coacervate combination in applications such as improving host responses to biosensors, implants and indwelling catheters, ${ }^{12,27}$ the use of ibuprofen in this study provides a model for other types of amphiphilic anionic drugs that might be encapsulated within PAH/TPP coacervates (e.g., diclofenac and valproate).

More broadly, this model system enables a more general analysis of how ionically crosslinked polymer networks interact with oppositely charged amphiphile (i.e., surfactant) molecules. Mixtures of oppositely charged surfactants and polyelectrolytes, which not infrequently appear in studies on new cosmetic and pharmaceutical formulations, ${ }^{28-33}$ can also self-assemble and undergo associative phase separation (either coacervation or precipitation). ${ }^{34-36}$ Thus, introduction of anionic surfactants into PAH/TPP mixtures may lead to competition between the anionic surfactant and TPP for the cationic PAH amine groups. Such competitive binding could affect both the coacervate formation and its payload uptake/release properties. Ibuprofen (at least in its sodium salt form) is a weak anionic surfactant with an aqueous critical micelle concentration (CMC) of roughly
$180 \mathrm{mM}^{37}$ and can therefore serve as a model system for the analysis of such effects.

To perform this study, the surfactant/polyelectrolyte interactions between ibuprofen and PAH (both with and without TPP) were first probed by isothermal titration calorimetry (ITC) and light scattering. The formation of ibuprofen-loaded coacervates was then characterized by visual observation and rheology, and their encapsulation properties were determined by UV-vis spectroscopy. UV-vis spectroscopy was then also used to analyze the ibuprofen release kinetics, using both $1 \times$ phosphate buffered saline (PBS) and deionized water as the release media (while also analyzing the PAH/TPP network stability by gravimetry). Finally, recognizing that ibuprofen is a relatively weak anionic surfactant, effects of stronger surfactants sodium dodecyl sulfate (SDS; CMC $=8 \mathrm{mM}^{38}$ ) and sodium sodium dodecylbenzenesulfonate (SDBS; $\mathrm{CMC}=3 \mathrm{mM}^{39}$ ) - on $\mathrm{PAH} / \mathrm{TPP}$ coacervation were also briefly explored.

\section{Materials and methods}

\subsection{Materials}

All experiments were conducted using Millipore Direct-Q 3 deionized water with an $18.2 \mathrm{M} \Omega \mathrm{cm}$ minimum resistivity. PAH (nominal molecular weight $=120-200 \mathrm{kDa}$ ) was purchased from Alpha Aesar (Ward Hill, MA). Ibuprofen and TPP were obtained from Sigma-Aldrich (St. Louis, MO), while $\mathrm{HCl}, \mathrm{NaOH}$ and PBS powder were purchased from Fisher Scientific (Fair Lawn, NJ). The SDS and SDBS were obtained from MP Biomedicals (Solon, OH) and TCI America (Portland, OR), respectively. All materials were used as received.

\subsection{Analysis of $\mathbf{P A H} / \mathrm{ibuprofen}$ interactions}

Surfactant/polyelectrolyte binding between anionic ibuprofen and cationic PAH was analyzed via ITC and light scattering. ITC measurements were performed at $25^{\circ} \mathrm{C}$ using a MicroCal VP-ITC instrument (Malvern, Northampton, MA). Fifteen mM aqueous ibuprofen solutions were titrated into aqueous PAH solutions, which had either 5, 10 or $30 \mathrm{mM}$ monomer concentrations. Both the ibuprofen and PAH solutions were adjusted to $\mathrm{pH} 7.0$ using either $\mathrm{NaOH}$ or $\mathrm{HCl}$ prior to the titrations. Moreover, to test the effect of TPP binding on the PAH/ibuprofen interactions, additional ITC experiments were performed where the $30 \mathrm{mM} \mathrm{PAH}$ solutions were replaced with soluble TPP/PAH complexes. These complexes were prepared at $0.2: 1$ and $0.4: 1 \mathrm{TPP}: \mathrm{PAH}$ molar ratios by titrating either $200 \mu \mathrm{l}$ or $400 \mu \mathrm{l}$ of $100 \mathrm{mM}$ TPP into $15 \mathrm{ml}$ of $30 \mathrm{mM}$ PAH solution (in $50 \mu \mathrm{l}$ aliquots with 5 min intervals 
between each addition). After equilibrating the final mixtures for $1 \mathrm{~h}$, the binding of ibuprofen to TPP-complexed PAH was probed by ITC.

In each ITC experiment, PAH or PAH/TPP solutions were placed in the sample cell and the ibuprofen solution was placed in the injector. The titrations were then performed using twenty-seven injections (one $2 \mu$ injection followed by twentysix $10 \mu$ l ones) separated by $10 \mathrm{~min}$ equilibration intervals. To ensure uniform mixing within the sample cell, its contents were mixed with an impeller-shaped injector tip at a standard $307 \mathrm{rpm}$ speed. The raw data obtained from each titration (see ESI, Fig. S1 $\dagger$ ) was then integrated over time to generate the final isotherms. Furthermore, to isolate the heat of binding from the heat of ibuprofen dilution, control experiments that quantified the dilution heats were performed by titrating the ibuprofen solution into control PAH-free water at $\mathrm{pH}$ 7.0. The integrated dilution heat was then subtracted from the experimental data to obtain the final enthalpic $\mathrm{PAH} /$ ibuprofen binding signature.

To also analyze the $\mathrm{PAH} / \mathrm{ibuprofen}$ interactions via light scattering, similar titrations were repeated manually inside $20 \mathrm{ml}$ scintillation vials stirred at $800 \mathrm{rpm}$ with $12 \mathrm{~mm} \times 4 \mathrm{~mm}$ magnetic stir bars. Here, $200 \mu \mathrm{l}$ aliquots of $15 \mathrm{mM}$ ibuprofen solution were sequentially titrated into $10 \mathrm{ml}$ of 5-30 mM PAH solutions (and solutions of soluble PAH/TPP complexes), with 5 min time increments between each ibuprofen addition. After each ibuprofen addition, the light scattering intensity from the $\mathrm{PAH} /$ ibuprofen mixtures was measured (at the $173^{\circ}$ detector angle) using a Zetasizer Nano ZS (Malvern, UK) dynamic and electrophoretic light scattering instrument. Each ITC and light scattering experiment was performed in triplicate.

\subsection{Ibuprofen-free coacervate preparation}

The PAH/TPP coacervates were prepared by adding $22 \mathrm{ml}$ of $11.2 \mathrm{wt} \%$ (304 mM) TPP into $1000 \mathrm{ml}$ of $0.3 \mathrm{wt} \%$ (30 mM) PAH solution stirred with a $12 \mathrm{~mm} \times 4 \mathrm{~mm}$ cylindrical magnetic stir bar at $400 \mathrm{rpm}$. The TPP additions were performed in eleven $2 \mathrm{ml}$ aliquots with $5 \mathrm{~min}$ time intervals between each addition and produced PAH/TPP mixtures with 0.2 : 1 TPP : PAH molar ratios. Both $\mathrm{PAH}$ and TPP solutions were adjusted to $\mathrm{pH} 7.0$ using $\mathrm{HCl}$ or $\mathrm{NaOH}$ prior to their mixing. The resulting coacervates were then equilibrated for $72 \mathrm{~h}$ before further use.

\subsection{Analysis of ibuprofen uptake properties}

Ionically crosslinked coacervates containing ibuprofen were prepared by first complexing the PAH with the drug and then adding TPP solution to PAH/ibuprofen mixtures. PAH/ibuprofen mixtures were prepared by pre-dissolving 1-5 $\mathrm{mg} \mathrm{ml}^{-1}$ (4.9-24.3 $\mathrm{mM}$ ) of ibuprofen in $500 \mathrm{ml}$ of water, whereupon 0.3 or $1.0 \mathrm{wt} \%$ $\mathrm{PAH}$ (30 or $107 \mathrm{mM}$ in its monomer units) was dissolved. To fully dissolve the ibuprofen (which was originally in its protonated form), the $\mathrm{pH}$ of its dissolution medium was raised to 12.5, which was well above its $\mathrm{p} K_{\mathrm{a}}(\sim 4.5-4.6) .{ }^{40}$ Solid PAH was then added to these ibuprofen solutions while, to ensure complete PAH dissolution, maintaining the $\mathrm{pH}$ above 12 (where the $\mathrm{PAH}$ remained largely deprotonated) by adding $1 \mathrm{M} \mathrm{NaOH}-$ i.e., to prevent $\mathrm{PAH} /$ ibuprofen complexation and precipitation during PAH addition.
On complete dissolution of the $\mathrm{PAH}$, the mixture $\mathrm{pH}$ was adjusted to $\mathrm{pH} 7.0$ using $\mathrm{HCl}$, whereupon insoluble $\mathrm{PAH} /$ ibuprofen complexes formed. These mixtures were equilibrated for $5 \mathrm{~h}$ (while stirring at $300 \mathrm{rpm}$ ) prior to TPP addition. Then, to form the coacervates, $5.25 \mathrm{ml}$ of $11.2 \mathrm{wt} \%$ (304 mM) TPP were added in eleven $1 \mathrm{ml}$ aliquots to generate $\mathrm{PAH} / \mathrm{TPP} / \mathrm{ibuprofen}$ mixtures with 0.2 : 1 TPP : PAH molar ratios.

After equilibrating the resulting coacervates for $72 \mathrm{~h}$, the supernatant phase was collected and analyzed for its ibuprofen content by UV-vis spectroscopy (at $\lambda=263 \mathrm{~nm}$ where $\varepsilon=$ $367.3 \mathrm{M}^{-1} \mathrm{~cm}^{-1}$ ). These measurements were performed using a Cary 50 UV-vis spectrophotometer (Sparta, NJ) and enabled the loading efficiency (LE) and loading capacity (LC) to be determined using the following equations:

$$
\begin{aligned}
& \mathrm{LE}=\left(1-\frac{C_{\mathrm{S}} V_{\mathrm{S}} M_{\mathrm{W}}}{W_{\mathrm{D}}}\right) \times 100 \% \\
& \mathrm{LC}=\frac{W_{\mathrm{D}}-C_{\mathrm{S}} V_{\mathrm{S}} M_{\mathrm{W}}}{W_{\mathrm{C}}} \times 100 \%
\end{aligned}
$$

where $C_{\mathrm{S}}$ was the (molar) supernatant ibuprofen concentration, $V_{\mathrm{S}}$ was the supernatant volume, $M_{\mathrm{W}}$ was the ibuprofen molecular weight, $W_{\mathrm{D}}$ was the total weight of added (ibuprofen) drug and $W_{\mathrm{C}}$ was the weight of coacervate formed. Experiments at each uptake condition were reproduced three times.

\subsection{Rheology}

Dynamic rheology of amphiphilic cargo-loaded PAH/TPP coacervates was characterized at room temperature using a Rheometric Scientific RDA III (Piscataway, NJ) strain-controlled rheometer fitted with $25 \mathrm{~mm}$ parallel plates. Amphiphilebearing PAH/TPP coacervates (formed in the presence of $24.3 \mathrm{mM}$ of either ibuprofen, SDS or SDBS) were prepared as described in Section 2.4 and compared with control samples that contained no payload. The resulting coacervates were equilibrated for $72 \mathrm{~h}$ prior to being loaded into the rheometer (in roughly $0.1 \mathrm{~g}$ quantities). The samples were then compressed to a $0.5 \mathrm{~mm}$ gap thickness, and allowed to relax until the normal force was below $100 \mathrm{~g}$. Excess coacervate was then removed using a razor blade and water was applied to the sides of the plates to keep the coacervate hydrated. After a strain amplitude sweep was conducted to determine the linear viscoelastic region, frequency sweeps were conducted at a $1.0 \%$ strain amplitude and 0.1-500 $\mathrm{rad} \mathrm{s}^{-1}$ angular velocities. Each rheological measurement was performed in triplicate.

\subsection{Analysis of ibuprofen release rates}

To analyze the ibuprofen release rates, ibuprofen-loaded coacervates were prepared and equilibrated for $72 \mathrm{~h}$ as described in Section 2.4. A known weight (roughly 0.1-0.2 g) of the coacervate was then placed into a $2 \mathrm{ml}$ microcentrifuge tube and covered with $1 \mathrm{ml}$ of the supernatant solution, which was obtained upon coacervation. This mixture was then centrifuged at $15000 \mathrm{rpm}$ for 90 min in an Eppendorf 5424 centrifuge (Hauppauge, NY) to flatten the surfaces of the $\mathbf{9}-\mathbf{1 0} \mathrm{mm}$ thick coacervate layers. After centrifugation, the supernatant was decanted and the 
(a)

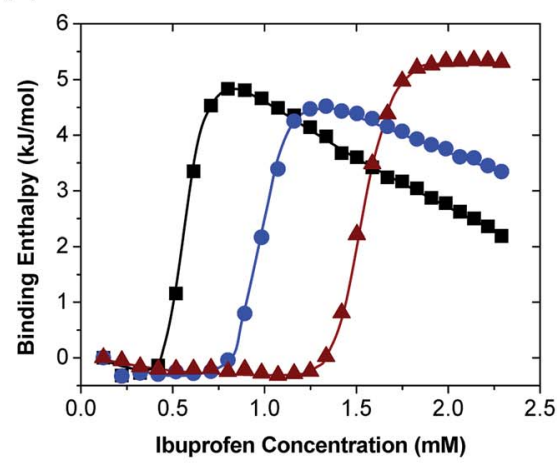

(c)

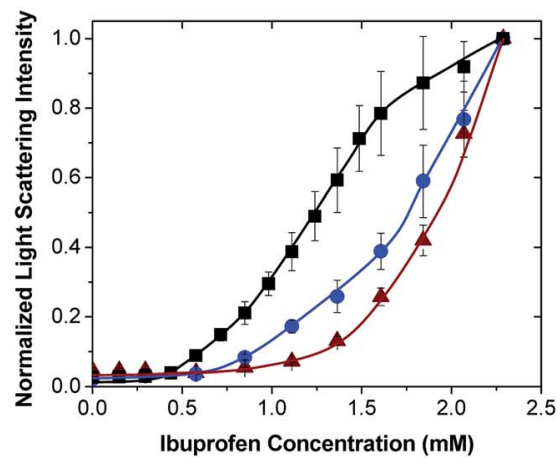

(b)

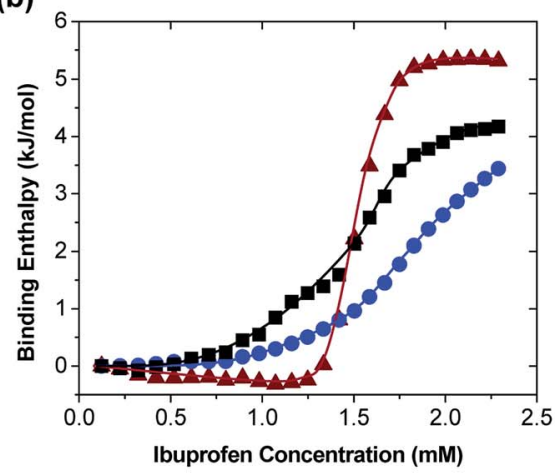

(d)

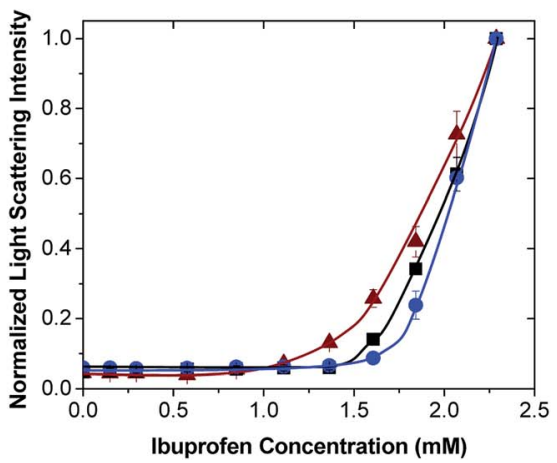

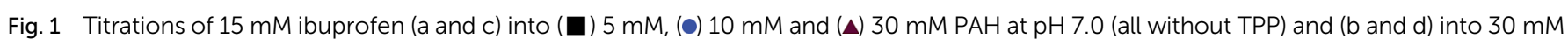
PAH mixed with TPP at (A) $0.00: 1,(\boldsymbol{\square}) 0.20: 1$ and (•) $0.40: 1$ TPP : PAH molar ratios analyzed by (a and b) ITC and (c and d) light scattering. The error bars are standard deviations and the lines are guides to the eye.

centrifuge tube and coacervate surface were wiped dry with a Kimwipe ${ }^{\mathrm{TM}}$ tissue. One $\mathrm{ml}$ of release media (either DI water or $1 \times$ PBS) was subsequently added, and release experiments were performed in an Eppendorf Thermomixer at $37{ }^{\circ} \mathrm{C}$ and a $400 \mathrm{rpm}$ mixing speed. The concentration of the ibuprofen released from the coacervates was measured over time by UV-vis spectroscopy (where $\varepsilon$ was the same as that in Section 2.4) and the release media was replaced (with fresh media) each time a measurement was taken. Two replicate samples were analyzed at each release condition.

\section{Results and discussion}

\section{1. $\mathbf{P A H / i b u p r o f e n ~ i n t e r a c t i o n s ~}$}

The binding of ibuprofen to the PAH was probed using ITC where the ibuprofen was titrated into variably concentrated (5$30 \mathrm{mM}$ ) PAH solutions at pH 7.0 (see Fig. 1a). The binding heat was initially minimal but, once a critical ibuprofen concentration was reached, increased sharply. This sharp rise was followed by either a gradual reduction (black squares and blue diamonds) or a plateau (red triangles) in the enthalpic signal. The sudden increase in binding heats within each thermogram reflected the critical aggregation concentration (CAC) for the ibuprofen/PAH binding, which increased from about 0.5 to $1.3 \mathrm{mM}$ as the PAH monomer concentration was raised from 5 to $30 \mathrm{mM}$ (Fig. 1a). This increase in the CAC with the polymer concentration was typical for surfactant binding to oppositely charged polyelectrolytes, ${ }^{\mathbf{4 1 , 4 2}}$ and might have reflected the elevated ionic strengths at higher polymer concentrations. ${ }^{41}$ At higher ibuprofen concentrations, however (at least at the two lower $\mathrm{PAH}$ concentrations), the binding enthalpy decreased again; evidently due to a gradual saturation of ibuprofen binding sites (i.e., the amine groups) on the PAH. ${ }^{42}$

To start analyzing how PAH/ibuprofen binding was affected by TPP addition during coacervate preparation, $\mathrm{PAH} / \mathrm{ibuprofen}$ interactions were also probed using $30 \mathrm{mM}$ PAH solutions with TPP added in either $0.00: 1,0.20: 1$ or $0.40: 1$ TPP : PAH molar ratios (see Fig. 3b). The CAC transition, which at the $0.00: 1$ TPP : PAH ratio was marked by a sharp increase in binding heat at roughly $1.3 \mathrm{mM}$ ibuprofen, became significantly more gradual with TPP addition, thus indicating a decrease in PAH/ ibuprofen binding cooperativity. ${ }^{\mathbf{4 2 , 4 3}}$ This decrease in cooperativity likely reflected a partial neutralization of cationic $\mathrm{PAH}$ amine groups by the strongly-binding $\mathrm{TPP},{ }^{44}$ which reduced the abundance of neighboring ibuprofen-accessible amine groups (thus frustrating cooperative hydrophobic interactions between the PAH/bound ibuprofen molecules). Since cooperative hydrophobic interactions are critical to strong surfactant/ polyelectrolytes binding, ${ }^{\mathbf{4 3 , 4 5}}$ this reduction in cooperativity suggests that TPP addition might weaken PAH/ibuprofen association.

The above interpretations of the ITC data were supported by the variations in normalized light scattering that occurred upon 

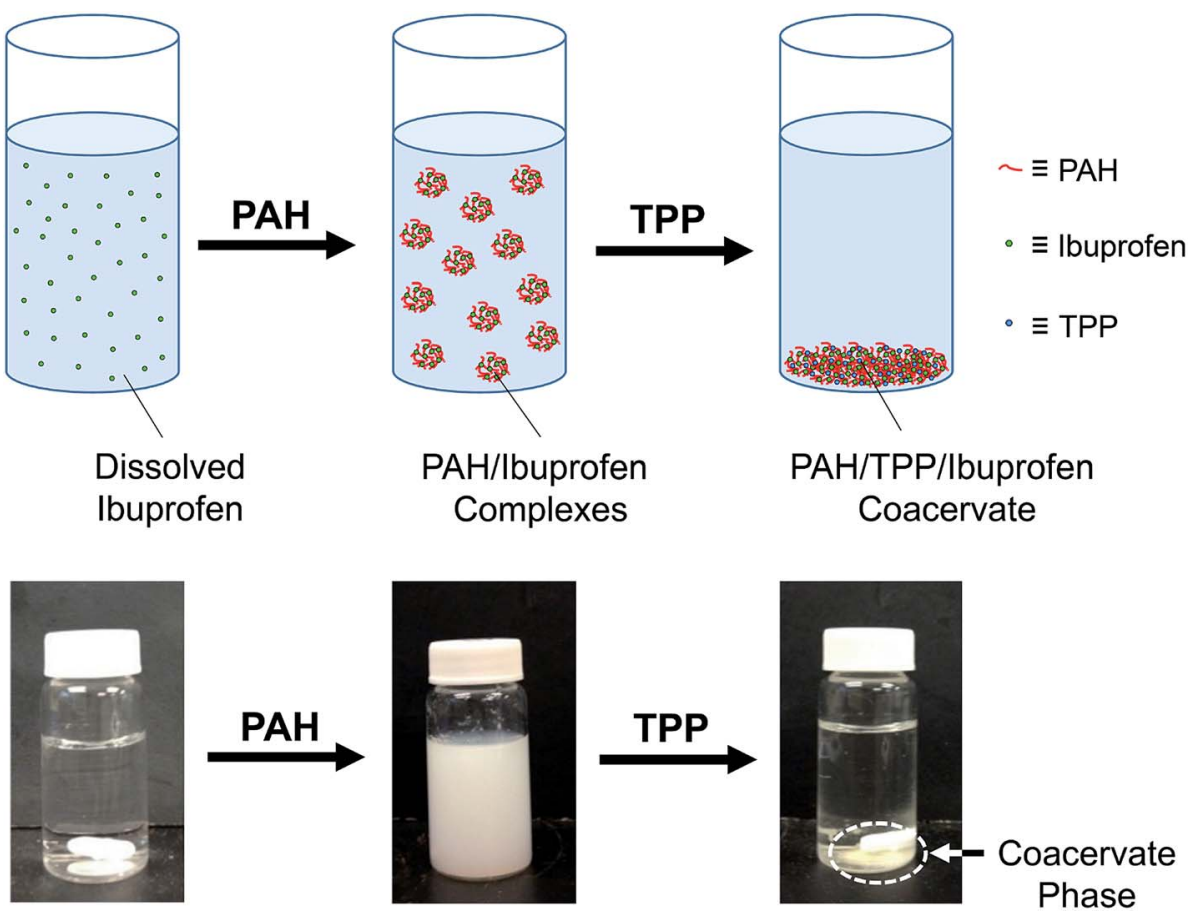

Fig. 2 Schematic drawings and digital photographs of the formation of ibuprofen-loaded PAH/TPP coacervates.

ibuprofen addition to PAH and PAH/TPP solutions. Here, the CAC transitions were indicated by sharp increases in light scattering, caused by the formation of insoluble colloidal PAH/ ibuprofen complexes. Consistent with the ITC data, these transitions shifted to higher ibuprofen concentrations (which were closely matched to those obtained by ITC) at higher PAH concentrations (Fig. 1c). Similarly, the sharp rise in the normalized light scattering intensity shifted to slightly higher ibuprofen concentrations upon TPP addition (Fig. 1d). This was despite the partial neutralization of the PAH with TPP (and the fact that the $0.40: 1$ TPP : PAH molar ratio was nearly high enough to induce insoluble complex formation even without ibuprofen $^{6}$ ) and supported the view that TPP weakens PAH/ ibuprofen binding. Since TPP is not expected to associate with the similarly charged ibuprofen molecules, this weakened PAH/ ibuprofen binding is attributed to PAH/TPP association, which (by reducing the availability of free PAH and TPP ionic groups) inhibits the interaction of both PAH and TPP with other solutes.

\subsection{Formation of ibuprofen-loaded coacervates}

When ibuprofen was added to $\mathrm{pH} 12.5$ water, the $\mathrm{pH}$ initially dropped to roughly 10.5-11.5 (10.5 for the highest, $24.3 \mathrm{mM}$ ibuprofen concentration and 11.5 for the lowest, $4.9 \mathrm{mM}$ ibuprofen concentration). This $\mathrm{pH}$ reduction occurred because the ibuprofen was initially in its protonated form and behaved as an acid. Prior to PAH addition, the $\mathrm{pH}$ of this ibuprofen solution was adjusted to and maintained above 12.0 (to keep the $\mathrm{PAH}$ deprotonated during dissolution). This was required because the $\mathrm{PAH}$ dissolution led to a reduction in $\mathrm{pH}$ and resulted in $\mathrm{PAH} /$ ibuprofen phase separation, which was postulated to interfere with further PAH dissolution. Hence, to ensure complete $\mathrm{PAH}$ dissolution, the $\mathrm{pH}$ was adjusted to 12.0 during the $\mathrm{PAH}$ addition/dissolution process. Once the $\mathrm{PAH}$ was fully dissolved, the $\mathrm{pH}$ of this mixture was adjusted to 7.0, which was below the $\mathrm{p} K_{\mathrm{a}}$ of $\mathrm{PAH}(\sim 8.7)^{46}$ and caused the amine groups to protonate and ionize. This PAH ionization resulted in colloidal dispersions due to the association between the cationic $\mathrm{PAH}$ and the anionic ibuprofen (see Fig. 2), which was wellestablished through the calorimetric and light scattering measurements in Fig. 1. These dispersions were not fully stable and formed small amounts of flaky, macroscopic precipitates. The subsequent addition of TPP to these PAH/ibuprofen dispersions resulted in the formation and aggregation of TPP/ $\mathrm{PAH}$ complexes (Fig. 2), where flaky $\mathrm{PAH} /$ ibuprofen precipitates were converted into ibuprofen-loaded PAH/TPP coacervates. $^{6,7}$ This conversion likely reflected the strong PAH/TPP binding, which not only contributed to the high stability of the PAH/TPP network (vide infra), but also displaced the weakerbinding ibuprofen molecules from the PAH chains.

\subsection{Ibuprofen encapsulation within PAH/TPP networks}

The formation of PAH/TPP coacervates in ibuprofen-containing mixtures resulted in ibuprofen encapsulation. The uptake of ibuprofen into these coacervates was greatly facilitated by the association of ibuprofen with PAH prior to the TPP addition and, because the $\mathrm{PAH} / \mathrm{ibuprofen}$ binding was cooperative (see Section 3.1), both the LE and LC increased with the added ibuprofen concentration (Fig. 3a and b). When $0.3 \mathrm{wt} \% \mathrm{PAH}$ solution was used in the encapsulation procedure, for instance (blue circles), LE increased from roughly 3\% when coacervation occurred in the presence $4.9 \mathrm{mM}$ ibuprofen to roughly $35 \%$ when coacervation occurred in the presence of $24.3 \mathrm{mM}$ 
(a)

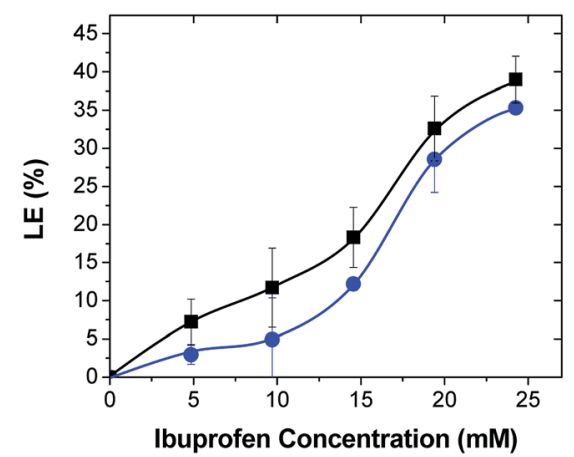

(b)

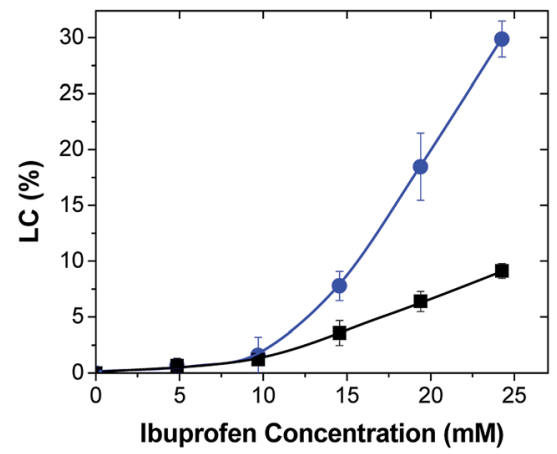

Fig. 3 Drug concentration effects on the (a) LE and (b) LC of ibuprofen uptake into PAH/TPP coacervates achieved at PAH (•) 0.3 and ( $\mathbf{\square}) 1.0$ wt\% $\mathrm{PAH}$ concentrations. The error bars are standard deviations while the lines are guides to the eye.

ibuprofen (Fig. 3a). Even more striking was the impact of ibuprofen concentration on the LC, which increased from less than $1 \%$ to roughly $30 \%$ upon the same increase in the initial ibuprofen concentration (Fig. 3b).

These high LC-values greatly exceeded those reported in the previous study on payload uptake and release using PAH/PPi and PAH/TPP coacervates where Fast Green FCF (FG) and Rhodamine B (RhB) dyes were used as model molecules and LCs did not exceed $3 \% .^{12}$ These differences in LC-values were because the dyes and PAH in the previous work were mixed in much lower payload : PAH ratios and, in the case of $\mathrm{RhB}$ (whose LC-values were even much lower than those of FG) reflected a lack of affinity between the payload and PAH. The superior uptake properties demonstrated with ibuprofen above, however, reflected a favorable PAH/ibuprofen affinity. In other words, though the PAH/ibuprofen binding was sufficient to concentrate the ibuprofen near the PAH chains (which enabled the high LC-values), it was substantially weaker than the PAH/ TPP binding, and thus allowed the TPP to efficiently displace ibuprofen from the PAH amine groups to form the ionic network. This ability of TPP to displace the PAH-bound ibuprofen enabled high ibuprofen : $\mathrm{PAH}$ ratios to be used during the encapsulation process, and thus gave rise to the very high LC-values without disrupting the ionic network formation.
To further confirm that ibuprofen did not disrupt the PAH/ TPP complexation, coacervates with and without ibuprofen loaded at the highest (roughly $30 \%$ ) LC-value were probed via dynamic rheology. For PAH/TPP coacervates without ibuprofen, the storage modulus, $G^{\prime}$, exceeded the loss modulus, $G^{\prime \prime}$, over the entire frequency range used (see Fig. 4a). This trend was consistent with previous rheological analysis of PAH/TPP networks formed from PAH and TPP solutions at neutral $\mathrm{pH}-$ levels and without added salt. ${ }^{6,7}$ Similarly, although the addition of concentrated ibuprofen to these coacervates brought the $G^{\prime}$ and $G^{\prime \prime}$ closer together, this nearing effect was mediated by a multifold increase in $G^{\prime \prime}$ rather than a decrease in $G^{\prime}$. Indeed, the $G^{\prime}$ also increased slightly with the addition of ibuprofen and remained above $G^{\prime \prime}$ over the entire experimental frequency range. Thus, the gel-like properties of the coacervates were largely preserved. These rheological trends suggest that, when weakly amphiphilic anionic payloads such as ibuprofen are used, the PAH/TPP coacervate networks can accommodate remarkably high (up to $30 \mathrm{wt} \%$ of the total network mass) drug. loadings, which are uncommon for hydrogel-based, long-term sustained release systems for water-soluble small molecules. ${ }^{19,23}$

While the LE was greatly enhanced at higher ibuprofen concentrations (see Fig. 3a), increasing the PAH concentration to $1 \mathrm{wt} \%$ produced only marginal improvements in LE-values, (a)

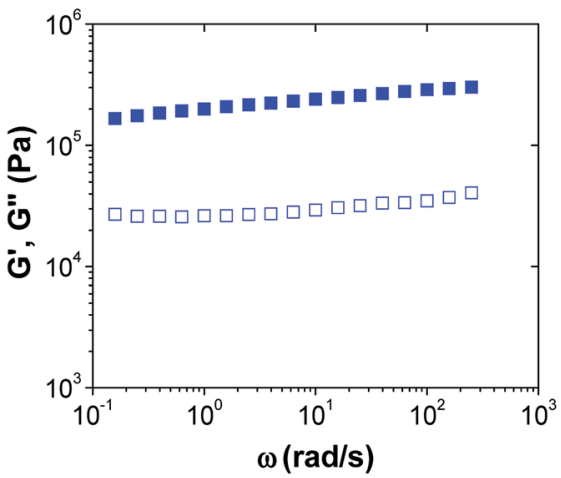

(b)

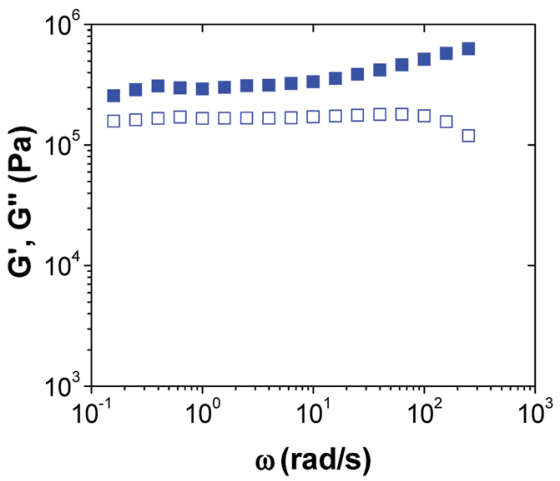

Fig. 4 Dynamic rheology of PAH/TPP coacervates comparing ( $\square$ ) $G^{\prime}$ and $(\square) G^{\prime \prime}$ for (a) ibuprofen-free coacervates and (b) coacervates formed in the presence of $24.3 \mathrm{mM}$ ibuprofen. Both coacervate types were analyzed after $72 \mathrm{~h}$ of equilibration in the mixtures from which they formed. 
(a)

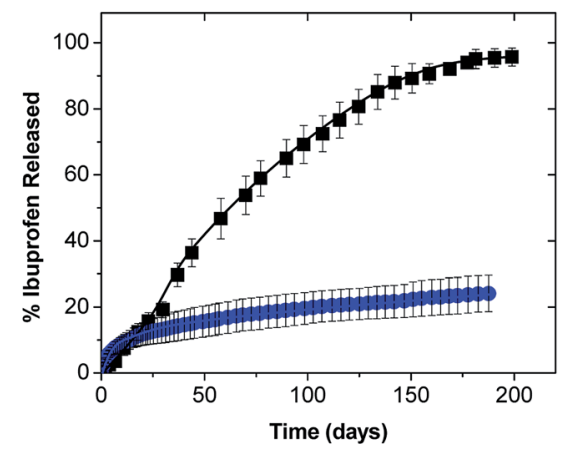

(c)

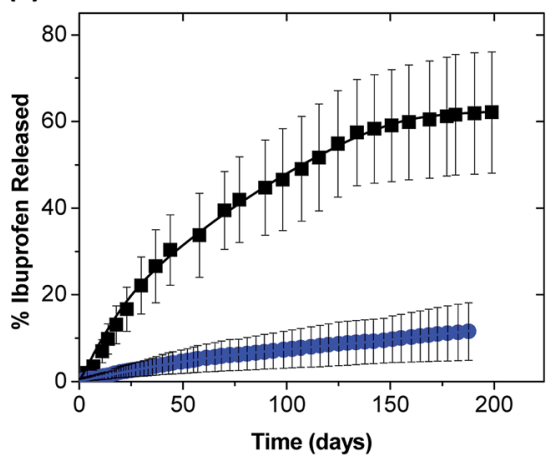

(b)

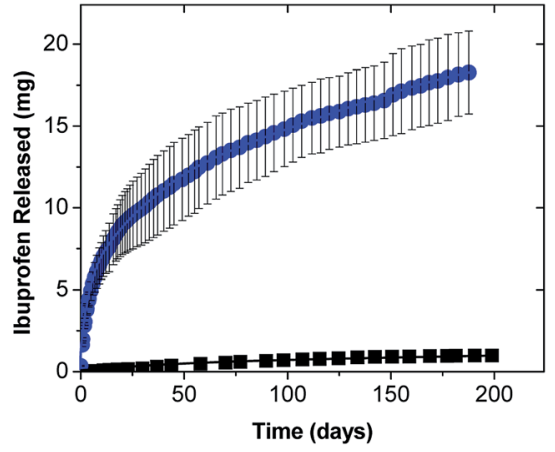

(d)

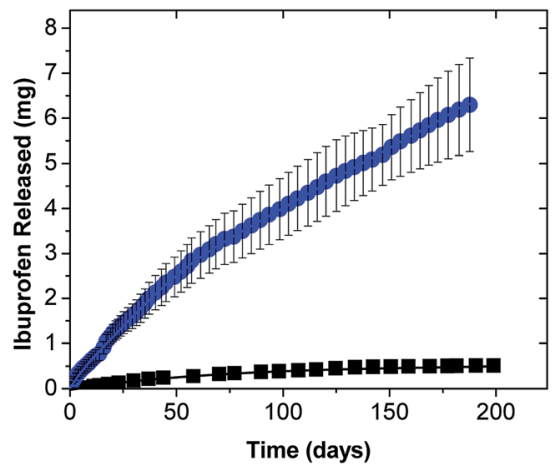

Fig. 5 Ibuprofen release profiles from PAH/TPP coacervates loaded using ( $\mathbf{\square}) 4.9$ and (•) $24.3 \mathrm{mM}$ initial ibuprofen concentrations, 0.3 wt\% PAH, and ( $a$ and b) $1 \times$ PBS and (c and d) DI water as the release media. The release profiles are plotted in terms of the ( $a$ and $c)$ percent or (b and d) total mass of the ibuprofen released. The error bars are standard deviations and the lines are a guide to the eye.

which were most significant at lower ibuprofen concentrations, but became very weak at the more-favorable 19.4-24.3 mM ibuprofen concentrations (Fig. 3a). This remarkably weak effect of increasing the PAH concentration might have reflected the cooperativity of the PAH/ibuprofen binding, which was essential for efficient ibuprofen uptake. Though adding more PAH increased the availability of ibuprofen binding sites, increased PAH : ibuprofen ratios both diminished the probability of favorable, cooperative interactions between PAH-bound ibuprofen molecules, and (for a constant ibuprofen concentration) increased the ionic strength. Indeed, as the parent PAH concentration was increased from 0.3 to $1 \mathrm{wt} \%$, the theoretical overall $\mathrm{NaCl}$ concentration (achieved under the assumption of complete ionic association between the ionizable PAH and TPP groups) increased from 30 to $107 \mathrm{mM}$. Accordingly, as shown in Fig. 1a, higher PAH : ibuprofen concentrations weakened PAH/ ibuprofen binding.

Furthermore, by distributing the encapsulated ibuprofen over a greater coacervate mass, this increase in PAH concentration sharply reduced the LC-values which, when the (most favorable) $24.3 \mathrm{mM}$ ibuprofen is used, dropped from around $30 \%$ to below $10 \%$. These PAH concentration effects are qualitatively consistent with those seen previously with the uptake of the cooperatively binding FG food coloring dye (though the LC-values for FG were much lower), ${ }^{12}$ and are likely general to other cooperatively binding anionic payloads. They also indicate that, to achieve the very high loadings (i.e., very high LC-values) of weakly amphiphilic payloads within these coacervates, low PAH concentrations should be used.

Besides tailoring the PAH concentration, PAH/TPP coacervates can be prepared using different $\mathrm{PAH}$ molecular weights (data not shown). Since surfactant/polyelectrolyte binding (which enables the high ibuprofen uptake), however, is only sensitive to polyelectrolyte molecular weight when the polymer chains are very short (with degrees of polymerization well below $100),{ }^{47,48}$ variations in PAH chain length are not expected to strongly affect uptake performance. In other words, the high LCvalues obtained herein can likely be achieved using an array of PAH molecular weights.

\subsection{Ibuprofen release from PAH/TPP coacervates}

Release of ibuprofen from coacervates loaded with either the lowest $(4.9 \mathrm{mM})$ or the highest $(24.3 \mathrm{mM})$ initial ibuprofen concentrations was then characterized using $1 \times$ PBS and DI water as the two model release media. At the lower ibuprofen loading where, based on the LC-values in Fig. $3 \mathrm{~b}$, the coacervates contained only $0.66 \pm 0.18 \mathrm{wt} \%$ ibuprofen, almost all of the payload was released into PBS over about 175 days (black squares in Fig. 5a). Conversely, coacervates prepared at the highest initial ibuprofen concentration (which contained $29.9 \pm$ $1.6 \mathrm{wt} \%$ ibuprofen), released only about $20 \%$ of the payload over 


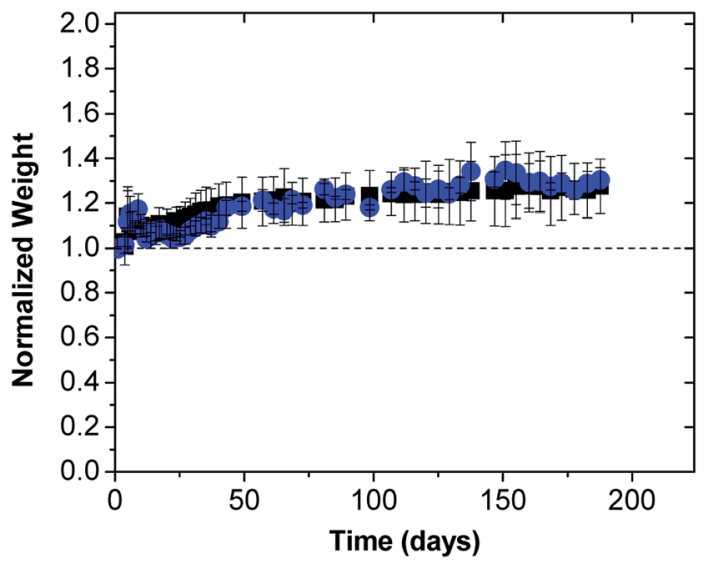

Fig. 6 Gravimetric analysis of the stability of PAH/TPP coacervates loaded with $24.3 \mathrm{mM}$ ibuprofen and $0.3 \mathrm{wt} \%$ PAH during drug release into ( $\mathbf{\square})$ DI water and ( $\bullet$ ) $1 \times$ PBS. The error bars are standard deviations while the dashed line indicates the initial coacervate weight.

the 200 day experiment (blue circles in Fig. 5b). Despite this lower fractional release, however, the coacervates with higher LC-values released a much higher total ibuprofen mass than their lower-LC counterparts (see Fig. 5b). Similar LC effects on the release rates were obtained when the PBS release medium was replaced by DI water (see Fig. $5 \mathrm{c}$ and d), though the release rates in this lower-ionic-strength release medium became substantially lower.

The multiple-month release achieved using the PAH/TPP coacervates was (aside from their low solute permeability) mediated by their high stability. Indeed, besides an initial reduction in coacervate opacity (which occurred during the initial weeks of the release process and likely reflected the loss of larger pores/defects in the polymer network ${ }^{49}$ ) and roughly a $20 \%$ increase in coacervate weight - which for coacervates loaded using $24.3 \mathrm{mM}$ ibuprofen was obtained in both DI water and $1 \times$ PBS (Fig. 6) - the coacervate appearance remained unchanged throughout the nearly 200 day release experiment. This relative invariance in appearance and normalized weight indicates that, even with extremely high drug loadings (which are orders of magnitude higher than those studied previously ${ }^{\mathbf{1 2}}$ ), the PAH/TPP complexes preserve their stability.

Interestingly, despite the release being faster in the higherionic-strength PBS than in DI water ( $c f$. Fig. 5a and c), the coacervate weights in DI water and PBS are virtually identical (Fig. 6). This suggests that, in addition to depending on the hydrodynamic mesh size and water content within the ionic PAH/TPP networks, ${ }^{12}$ the release rates might depend on the ionic crosslink relaxation times, which diminish with increasing ionic strength. ${ }^{7}$ In other words, in $1 \times$ PBS the ionic crosslinks break and reform more rapidly, which facilitates ibuprofen diffusion through the dense ionic networks.

In some ways, the multiple-month ibuprofen release is similar to that seen in the previous work on sustained release from $\mathrm{PAH} / \mathrm{PPi}$ and $\mathrm{PAH} / \mathrm{TPP}$ coacervates, ${ }^{\mathbf{1 2}}$ where dye molecules were used as model payloads. The decrease in the rate of fractional drug release with the loaded ibuprofen concentration in
Fig. 5a and c, for instance, is qualitatively similar to that seen previously with the cooperatively-binding $\mathrm{FG}^{\mathbf{1 2}}$ and evidently reflects an increase in the cooperative ibuprofen association (with other ibuprofen molecules), which reduces its effective diffusion rate. The same parallel holds for the increase in the total payload mass released with the encapsulated payload concentration (in Fig. 5b and d) ${ }^{12}$ which again indicates that the rates of multiple-month payload delivery can be extensively tuned by varying its initial concentration within the coacervate.

Despite these similarities, however, the ibuprofen release profiles differ from those of FG in two key ways. First, the fractional release of ibuprofen is significantly more rapid than that obtained for FG (where less than 5\% of the payload was released into PBS over $80-160$ d). ${ }^{12}$ This difference in release kinetics may be attributed to differences in the size and flexibility of the two model payloads - i.e., FG is a relatively stiff, polyaromatic molecule with a molecular weight above $800 \mathrm{Da}$, while ibuprofen is a more flexible (and smaller) molecule with a molecular weight (in its sodium salt form) of only $228 \mathrm{Da}$. Due to this difference in molecular size and flexibility, ibuprofen was much more mobile within the ionic PAH/TPP network. This view was supported by ibuprofen release also being faster than that obtained for Rhodamine B (RhB; another larger, polyaromatic dye molecule), which was essentially non-binding to the PAH matrix, but still exhibited release properties that were similar to FG. ${ }^{12}$ Thus, the variability in release rates between ibuprofen and the previously studied dye molecules likely predominantly reflected differences in their molecular size and flexibility. Despite the faster release of ibuprofen, however, the release was sustained over more than 6 months, indicating that multiple-month release from PAH/TPP coacervates can be achieved even with smaller low-molecular-weight payloads (and not just the larger, polyaromatic small molecules such as the FG and RhB dyes).

The second key difference between ibuprofen and FG (and $\mathrm{RhB}$ ) dye release was that, because much more ibuprofen was loaded into the PAH/TPP networks than the dyes in the previous work, the PAH/TPP coacervates delivered ibuprofen in much larger quantities than the dyes in the previous study. Indeed, in the time required for them to release roughly $0.1 \mathrm{mg} F \mathrm{~F}$, the $\mathrm{PAH} / \mathrm{TPP}$ coacervates were able to release $10-20 \mathrm{mg}$ of ibuprofen. These much higher payload doses were achieved while sustaining the controlled release over multiple-month timescales and showed that, when weakly amphiphilic anionic payloads (such as ibuprofen) are used, the highly sustained release can be achieved with a wide range of drug doses.

\subsection{Effect of amphiphile strength}

Since successful PAH/TPP coacervate formation depended on the efficient displacement of ibuprofen from PAH by strongerbinding TPP ions, ${ }^{12}$ we also hypothesized that the successful incorporation of ibuprofen within PAH/TPP coacervates might not extend to stronger anionic surfactants that bind more strongly to PAH. To this end, the encapsulation procedure used with weakly amphiphilic ibuprofen $\left(\mathrm{CMC}=180 \mathrm{mM}^{37}\right)$ was also attempted with strong anionic surfactants, SDS $(\mathrm{CMC}=$ 
(a)

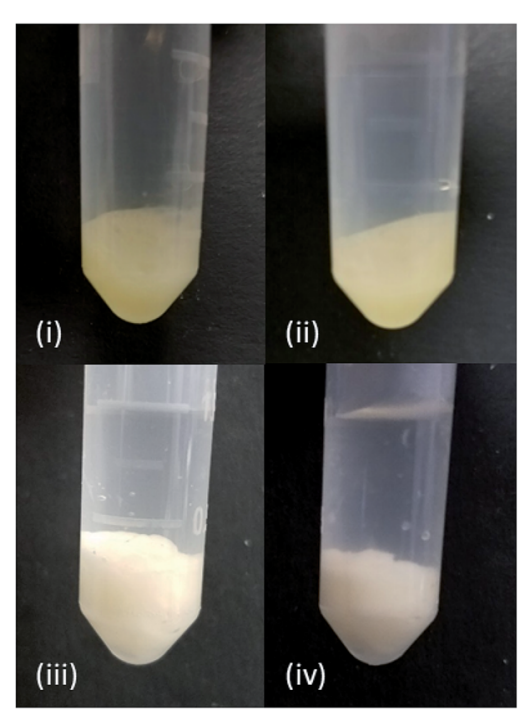

(b)

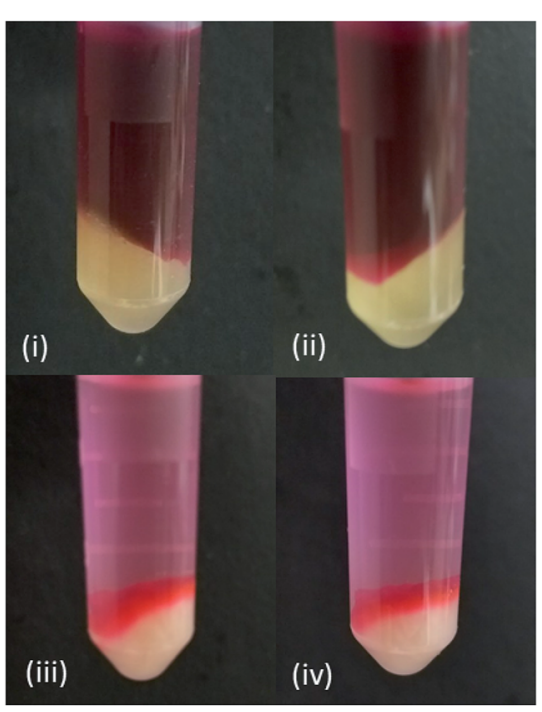

Fig. 7 Digital photographs of (a) PAH/TPP coacervates prepared in the presence of: (i) no payload, (ii) ibuprofen, (iii) SDBS and (iv) SDS (with each amphiphilic payload present at a concentration of $24.3 \mathrm{mM}$ ) and (b) the same PAH/TPP coacervates after $7 \mathrm{~d}$ of equilibration in the presence of $1.0 \mathrm{ml}$ of $2.1 \mathrm{mM}$ RhB dye.

$8 \mathrm{mM}^{38}$ ) and SDBS (CMC $=3 \mathrm{mM}^{39}$ ) Encapsulation of these strong surfactants produced several differences in PAH/TPP coacervate properties. First, unlike the colloidal PAH/ ibuprofen complexes that formed when the $\mathrm{pH}$ of $\mathrm{PAH} /$ ibuprofen mixtures was reduced to 7.0, stronger-bound PAH/ SDS and PAH/SDBS complexes rapidly formed macroscopic precipitates. This difference in properties persisted after the addition of TPP. Unlike the encapsulation of ibuprofen, which had minimal impact on PAH/TPP coacervate appearance and an enhancement in $G^{\prime}$ and $G^{\prime \prime}$-values (see Fig. 7ai and ii and 4), when SDS and SDBS where used, the coacervate became much more opaque (Fig. 7aiii and iv) with lower (especially at lower oscillation frequencies) $G^{\prime}$ and $G^{\prime \prime}$-values (see ESI, Fig. S2 $\dagger$ ).

Based on the higher opacities and lower $G^{\prime}$-values (which indicated larger pore size ${ }^{49}$ and weakened PAH/TPP crosslinking ${ }^{7}$ respectively), we hypothesized that the presence of strong anionic amphiphiles diminished the ionic crosslink densities within the coacervates and, thus, compromised their resistance to solute diffusion. To quickly test this, RhB dye, which was essentially non-binding to the PAH/TPP networks, ${ }^{12}$ was added (in a $2.1 \mathrm{mM}$ concentration) to the supernatant and allowed to diffuse into the coacervate for 7 days. In the case of the payload-free and ibuprofen-loaded coacervates, there was no visible RhB penetration into the PAH/TPP coacervates (with virtually all the red $\mathrm{RhB}$ dye remaining in the supernatant phase and no RhB visible in the coacervate; see Fig. 7bi and ii). Conversely, when the coacervates contained the strongerbinding SDS and SDBS, a significant portion of the coacervate turned red due to RhB penetration (Fig. 7biii and iv), thus confirming that the PAH/TPP networks became more permeable, likely due to a reduction in the TPP crosslinking of PAH chains. This disruption of PAH/TPP crosslinking likely reflects the fact that, due to the stronger surfactant/PAH binding, TPP is no longer able to displace the surfactant from the PAH chains.
Another interesting observation was that, though RhB did not preferentially partition into PAH/TPP coacervates, ${ }^{12}$ the SDS and SDBS-bearing PAH/TPP networks were able to take up most of the RhB from the supernatant (compare light pink supernatant phases in Fig. 7biii and iv with the dark red ones in Fig. 7bi and ii). Indeed, when samples in Fig. 7biii and iv were observed after 2 months, the supernatant phases were essentially clear (data not shown), suggesting nearly full partitioning of $\mathrm{RhB}$ into $\mathrm{PAH} / \mathrm{TPP} / \mathrm{SDS}$ and PAH/TPP/SDBS networks. This enhanced partitioning into the ionic networks might have reflected the hydrophobic interactions between aliphatic surfactant tails and aromatic RhB rings. Thus, though incorporation of strong anionic amphiphiles appears to weaken the diffusion barrier properties of PAH/TPP networks, it might in some cases be beneficial in either enhancing payload uptake properties (for drugs exhibiting little natural affinity for PAH/TPP networks) and, as has recently been explored with other coacervates systems, ${ }^{50,51}$ enhance the use of these ionic networks as adsorbents. Similarly, strong anionic surfactants may be useful in tailoring release rates from PAH/TPP coacervates in cases where the very slow release from these materials needs to be accelerated.

\subsection{Further discussion}

Overall, we have shown that gel-like PAH/TPP coacervates combine extremely high loadings of weakly amphiphilic anionic payloads with their release over multiple-month timescales, which is very difficult to achieve with small, water-soluble molecules using other, gel-based (and gel-like) sustained release devices. Achieving multiple-week or -month delivery of such small, water-soluble payloads from hydrogels, for instance, typically requires techniques such as: (1) chemical attachment of the payload molecules to the hydrogel, which 
enables the long-term release rates to be controlled by the slow chemical cleavage rate $;^{20-22}$ (2) development of a scaffold that can physically bind the payload molecules, to retard their diffusion; ${ }^{19,23}$ and (3) encapsulation of the payload within colloidal structures (such as liposomes or polymeric micro- and nanostructures), which are dispersed within the gels and, due to their low payload permeability, control the release rates. ${ }^{\mathbf{1 4 - 1 9}}$ Each of these approaches, however, increases the complexity of the gel-like controlled release devices and typically generate LCvalues that are much lower than those reached in Fig. 3b. ${ }^{15,19,21,23}$ Though the high loading and multiple-month release are only demonstrated here with the potent anti-inflammatory drug, ibuprofen (which could potentially be released from PAH/TPP coacervates to improve the host response during the application of biosensors, implants or indwelling catheters ${ }^{12,27}$ ), these properties are also likely accessible with other weaklyamphiphilic and hydrotropic payloads whose use is a subject of ongoing studies. Further, the above experiments have shown that solute partitioning and permeability in PAH/TPP coacervates can be tailored through strong anionic amphiphile incorporation. This ability to achieve high cargo loadings, multiple-month release and tunable uptake/permeability, along with their promising in vitro cytocompatibility, ${ }^{12}$ could make $\mathrm{PAH} / \mathrm{TPP}$ coacervates attractive for a broad range of pharmaceutical, household and even environmental applications.

\section{Conclusions}

PAH/TPP coacervates were able to encapsulate a weakly amphiphilic small molecule drug (ibuprofen) with extremely high LC-values (where the ibuprofen comprised up to $30 \%$ of the total coacervate mass) and then release this drug over timescales exceeding 6 months. This unusual combination of properties likely reflected: (1) the affinity of the anionic amphiphile for the oppositely charged $\mathrm{PAH}$, which localized the payload near the polymer chains; and (2) the ability of the stronger-binding TPP to efficiently displace the payload from the cationic amine groups and form densely crosslinked ionic networks. Highest LC-values were achieved at high ibuprofen and low PAH concentrations. The release rates, on the other hand, could be readily tuned by varying the amount of ibuprofen initially loaded into the PAH/TPP coacervates. Overall, these findings suggest that gel-like PAH/TPP coacervates could be attractive for the multiple-month delivery of weakly amphiphilic drugs.

In contrast to the weakly amphiphilic ibuprofen, incorporation of stronger anionic amphiphiles (SDS and SDBS) appeared to interfere with the formation of PAH/TPP crosslinks. This in turn made the PAH/TPP complexes more permeable to small molecules and caused significant diffusion through PAH/ TPP networks over the (significantly shorter) timescale of days. The presence of these strong amphiphiles within the PAH/TPP networks, however, also impacted the coacervate affinity for other solutes (as demonstrated by their increased uptake of RhB dye). These abilities to enhance dye uptake and accelerate solute transport suggest that strong amphiphile incorporation within PAH/TPP coacervates could be utilized to tailor their payload uptake and release properties.

\section{Conflicts of interest}

Y. L. has a competing financial interest in a patent (US 9814778 B2) on the use PAH/TPP ionic networks in underwater adhesion and sustained release applications. The other authors have no conflicts of interest to declare.

\section{Acknowledgements}

The authors gratefully acknowledge the National Science Foundation (CBET-1133795, CBET-1150908 and IIP-1701104) for supporting this work and Ms. Sabrina S. Alam (Univ. of Toledo) for assisting with some of the experiments.

\section{References}

1 R. Bodmeier, H. Chen and O. Paeratakul, Pharm. Res., 1989, 6, 413-417.

2 H. G. Bungenberg de Jong, in Colloid Science, ed. H. R. Kruyt, Elsevier, Amsterdam, 1949, ch. X, vol. II, pp. 335-432.

3 A. Martinsen, G. Skjak-Braek and O. Smidsrod, Biotechnol. Bioeng., 1989, 33, 79-89.

4 V. S. Murthy, R. K. Rana and M. S. Wong, J. Phys. Chem. B, 2006, 110, 25619-25627.

5 H. G. Bagaria and M. S. Wong, J. Mater. Chem., 2011, 21, 9454-9466.

6 Y. Huang, P. G. Lawrence and Y. Lapitsky, Langmuir, 2014, 30, 7771-7777.

7 P. G. Lawrence and Y. Lapitsky, Langmuir, 2015, 31, 15641574.

8 R. K. Rana, V. S. Murthy, J. Yu and M. S. Wong, Adv. Mater., 2005, 17, 1145-1150.

9 J. Yu, V. S. Murthy, R. K. Rana and M. S. Wong, Chem. Commun., 2006, 1097-1099.

10 M. A. Yaseen, J. Yu, M. S. Wong and B. Anvari, Opt. Express, 2007, 16, 20577-20587.

11 J. Yu, D. Javier, M. A. Yaseen, N. Nitin, R. RichardsKortum, B. Anvari and M. S. Wong, J. Am. Chem. Soc., 2010, 132, 1929-1938.

12 P. G. Lawrence, P. S. Patil, N. D. Leipzig and Y. Lapitsky, ACS Appl. Mater. Interfaces, 2016, 8, 4323-4335.

13 E. M. Tavera, S. B. Kadali, H. G. Bagaria, A. W. Liu and M. S. Wong, AIChE J., 2009, 55, 2950-2965.

14 P.-C. Chen, Y. J. Park, L.-C. Chang, D. S. Kohane, R. H. Bartlett, R. Langer and V. C. Yang, J. Biomed. Mater. Res., Part A, 2004, 70A, 412-419.

15 I. Galeska, T. K. Kim, S. D. Patil, U. Bhardwaj, D. Chattopadhyay, F. Papadimitrakopoulos and D. J. Burgess, AAPS J., 2005, 7, E213-E240.

16 I. Lynch and K. A. Dawson, J. Phys. Chem. B, 2004, 108, 10893-10898.

17 I. Lynch, P. de Gregorio and K. A. Dawson, J. Phys. Chem. B, 2005, 109, 6257-6261. 
18 S. Mourtas, S. Fotopoulou, S. Duraj, V. Sfika, C. Tsakiroglou and S. G. Antimisiaris, Colloids Surf., B, 2007, 55, 212-221.

19 D. Sivakumaran, D. Maitland and T. Hoare, Biomacromolecules, 2011, 12, 4112-4120.

20 K. H. Bouhadir, G. M. Kruger, K. Y. Lee and D. J. Mooney, J. Pharm. Sci., 2000, 89, 910-919.

21 C. R. Nuttelman, M. C. Tripodi and K. S. Anseth, J. Biomed. Mater. Res., Part A, 2006, 76A, 183-195.

22 R. G. Schoenmakers, P. van de Wetering, D. L. Elbert and J. A. Hubbell, J. Controlled Release, 2004, 95, 291-300.

23 P. Andrade-Vivero, E. Fernandez-Gabriel, C. AlvarezLorenzo and A. Concheiro, J. Pharm. Sci., 2007, 96, 802813.

24 J. F. R. dos Santos, R. Couceiro, A. Concheiro, J. J. TorresLabandeira and C. Alvarez-Lorenzo, Acta Biomater., 2008, 4, 745-755.

25 T. R. Hoare and D. S. Kohane, Polymer, 2008, 49, 1993-2007.

26 D. Goldsmith, L. Scott, R. Cvetković and G. Plosker, Drugs, 2008, 68, 85-104.

27 M. S. Jaradat, B. Wongsud, S. Phornchirasilp, S. M. Rangwala, G. Shams, M. Sutton, K. J. Romstedt, D. J. Noonan and D. R. Feller, Biochem. Pharmacol., 2001, 62, 1587-1595.

28 P. Hossel, R. Dieing, R. Norenberg, A. Pfau and R. Sander, Int. J. Cosmet. Sci., 2000, 22, 1-10.

29 U. Kastner, H. Hoffmann, R. Donges and R. Ehrler, Colloids Surf., A, 1996, 112, 209.

30 S. Llamas, E. Guzman, F. Ortega, N. Baghdadli, C. Cazeneuve, R. G. Rubio and G. S. Luengo, Adv. Colloid Interface Sci., 2015, 222, 461-487.

31 J. Mirtič, K. Kogej, S. Baumgartner, G. Smistad, J. Kristl and M. Hiorth, Int. J. Pharm., 2016, 511, 774-784.

32 A. F. Thunemann and S. General, J. Controlled Release, 2001, 75, 237-247.

33 S. Zhou, C. Xu, J. Wang, P. Golas, J. Batteas and L. Kreeger, Langmuir, 2004, 20, 8482.

34 D. Li, M. S. Kelkar and N. J. Wagner, Langmuir, 2012, 28, 10348-10362.
35 L. Piculell, J. Norrman, A. V. Svensson, I. Lynch, J. S. Bernardes and W. Loh, Adv. Colloid Interface Sci., 2009, 147-148, 228-236.

36 A. F. Thunemann, M. Muller, H. Dautzenberg, J.-F. Joanny and H. Lowen, Adv. Polym. Sci., 2004, 166, 113-171.

37 A. Ridell, H. Evertsson, S. Nilsson and L. O. Sundelöf, J. Pharm. Sci., 1999, 88, 1175-1181.

38 J. Sjostrom and L. Piculell, Langmuir, 2001, 17, 3836.

39 Y. I. Gonzalez, M. Stjerndahl, D. Danino and E. W. Kaler, Langmuir, 2004, 20, 7053.

40 H. Potthast, J. Dressman, H. Junginger, K. Midha, H. Oeser, V. Shah, H. Vogelpoel and D. Barends, J. Pharm. Sci., 2005, 94, 2121-2131.

41 N. Jain, S. Trabelsi, S. Guillot, D. McLoughlin, D. Langevin, P. Letellier and M. Turmine, Langmuir, 2004, 20, 8496.

42 Y. Lapitsky, M. Parikh and E. W. Kaler, J. Phys. Chem. B, 2007, 111, 8379-8387.

43 P. Hansson and M. Almgren, J. Phys. Chem., 1996, 100, 9038-9046.

44 Y. Huang and Y. Lapitsky, J. Phys. Chem. B, 2013, 117, 9548-9557.

45 A. Malovikova, K. Hayakawa and J. C. T. Kwak, J. Phys. Chem., 1984, 88, 1930-1933.

46 S. R. Bhatia, S. F. Khattak and S. C. Roberts, Curr. Opin. Colloid Interface Sci., 2005, 10, 45-51.

47 J. Liu, K. Shirahama, T. Miyajima and J. Kwak, Colloid Polym. Sci., 1998, 276, 40-45.

48 J. Liu, N. Takisawa, K. Shirahama, H. Abe and K. Sakamoto, J. Phys. Chem. B, 1997, 101, 7520-7523.

49 F. Weinbreck, H. S. Rollema, R. H. Tromp and C. G. de Kruif, Langmuir, 2004, 20, 6389-6395.

50 M. Zhao, S. A. Eghtesadi, M. B. Dawadi, C. Wang, S. Huang, A. E. Seymore, B. D. Vogt, D. A. Modarelli, T. Liu and N. S. Zacharia, Macromolecules, 2017, 50, 3818-3830.

51 M. Zhao and N. S. Zacharia, Macromol. Rapid Commun., 2016, 37, 1249-1255. 